\title{
O treinamento aeróbio melhora o sono de estudantes universitórios?
}

\section{Aerobic exercise training improves sleep in college students?}

\author{
Thúlio Nilson do Nascimento Pereira'; Maria do Socorro Brasileiro-Santos²; Anna Myrna \\ Jaguaribe de Lima $^{3}$ \\ ${ }^{1}$ Departamento de Educação Física - Universidade Federal Rural de Pernambuco - UFRPE. Recife, PE - Brasil. \\ ${ }^{2}$ Departamento de Educação Física - Universidade Federal da Paraíba - UFPB. João Pessoa, PB - Brasil. \\ Departamento de Morfologia e Fisiologia Animal - Universidade Federal Rural de Pernambuco - UFRPE. Recife, PE - Brasil.
}

\author{
Endereço para Correspondência: \\ Anna Myrna Jaguaribe de Lima \\ Rua Manoel de Medeiros, s/n - Dois Irmãos \\ CEP: 52171-900- Recife, PE - Brasil \\ annamyrna@uol.com.br
}

\begin{abstract}
Resumo
Introdução: O sono é um estado fisiológico complexo e essencial. A qualidade do sono pode ser melhorada através da prática regular de exercício. Objetivo: Determinar a eficácia do treinamento aeróbio sobre a qualidade do sono (QS) e a sonolência diurna excessiva (SDE) em estudantes universitários. Métodos: 16 voluntários, divididos em grupo controle $(G C ; n=8)$ e grupo treinado $(G T ; n=8)$. O GT realizou 8 semanas de treinamento aeróbio e em ambos os grupos foram avaliados a QS, utilizando o índice de qualidade do sono de Pittsburgh (PSQI) e a SDE, através da escala de sonolência de Epworth (ESE). Resultados: O GT apresentou menor escore de PSQI comparado ao GC. Sobre a SDE, na comparação intragrupos, não houve alteração em nenhum dos grupos. Conclusão: De acordo com os resultados, o treinamento aeróbio de intensidade moderada melhora a qualidade do sono, porém parece não afetar a sonolência diurna excessiva.
\end{abstract}

Descritores: Sono; Distúrbios do Sono por Sonolência Excessiva; Exercício Aeróbio; Estudantes.

\begin{abstract}
Abstrac
Introduction: Sleep is an essential and complex physiological state. Sleep quality can be improved through regular exercise practice. Objective: To determine the effectiveness of moderate aerobic training on sleep quality (SQ) and excessive daytime sleepiness (EDS) in college students. Methods: 16 volunteers, divided in 2 groups: control group (CG; $\mathrm{n}=8$ ), no intervention, and training group (TG; $\mathrm{n}=$ 8 ) that performed 8 weeks of moderate aerobic training. Pittsburgh sleep quality index (PSQI) was used to evaluate SQ and Epworth sleepiness scale (ESS) was used to assess EDS. Results: TG showed lower PSQI scores compared to the CG. In addition, TG improved SQ, but there was no change in CG. Regarding EDS, in intragroup comparison, there was no change in both groups. Conclusion: According to the results, aerobic training of moderate intensity improves SQ, but does not seem to affect EDS.
\end{abstract}

Keywords: Sleep; Disorders of Excessive Somnolence; Aerobic Exercise; Students. 


\section{Introdução}

O sono é um estado funcional, reversível e cíclico, com manifestações comportamentais caracterizadas por imobilidade relativa e menor resposta aos estímulos externos, variações de parâmetros biológicos e de atividade mental ${ }^{1}$. O sono também é considerado um estado vital e complexo, caracterizado por processos ativos e altamente organizados ${ }^{2}$. Da Costa e Ceolim ${ }^{3}$ definiram o sono como um processo cíclico, composto por cinco estágios ou fases que se alternam e diferenciam-se, em termos fisiológicos, de acordo com o padrão das ondas cerebrais obtidas no eletroencefalograma (EEG) e a presença ou ausência de movimentos oculares rápidos, além de mudanças em outras variáveis como o tônus muscular e o padrão cardiorrespiratório. As proporções de cada estágio variam segundo a faixa etária.

A International Classification of Sleep Disorders (ICSD) versão 2 listou 81 distúrbios do sono principais, distribuídos em 8 grandes categorias: 1) insônias; 2) distúrbios do sono relacionados à respiração; 3) hipersonias de origem central; 4) distúrbios do sono relacionados ao ciclo circadiano; 5) parassonias; 6) distúrbios do sono relacionados ao movimento; 7) sintomas isolados; 8) outros distúrbios do sono ${ }^{4}$.

Sobre a quantidade de sono necessária, embora seja preciso dormir algumas horas a cada 24 horas, não é a quantidade e sim a qualidade do sono obtido nessas horas, associada à necessidade individual, que determinam a normalidade desta função ${ }^{5}$. A privação do sono, condição na qual o sono é inadequado, pode causar redução do desempenho, comprometimento da atenção e prejuízos à saúde em geral ${ }^{6}$. Além disso, também podem estar presentes sintomas como sonolência diurna excessiva, aumento da irritabilidade, alterações metabólicas, endócrinas, imunológicas, quadros hipertensivos, cansaço, náuseas, dores de cabeça, ardência nos olhos, visão turva, dores articulares e diminuição da libido ${ }^{7-10}$.
Um fator que pode afetar a qualidade do sono é prática regular de exercício físico. Embora o exercício físico seja aceito pela American Sleep Disorders Association (ASDA) como uma intervenção não-farmacológica para a melhoria do sono ${ }^{11}$, alguns estudos vem demonstrando o efeito benéfico do exercício físico sobre a qualidade do sono ${ }^{12,13}$. Um estudo realizado na cidade de São de Paulo fez um levantamento e demonstrou que entre 27,1 e $28,9 \%$ das pessoas fisicamente ativas e 72,9 e 71,1\% dos sedentários, se queixavam de insônia e sonolência diurna excessiva, respectivamente ${ }^{14}$.

A alta demanda acadêmica dos estudantes universitários pode causar alterações nos seus horários de descanso. Assim, a falta de organização de tempo pode levar esses estudantes a desenvolverem um comportamento sedentário que interferirá na qualidade do sono destes indivíduos. O exercício físico realizado de forma regular pode causar uma melhora na qualidade do sono e diminuição da sonolência diurna excessiva. Com isso, o objetivo do nosso estudo foi determinar a eficácia do treinamento físico aeróbio de intensidade moderada sobre a qualidade do sono e a sonolência diurna excessiva em estudantes universitários.

\section{Materiais e métodos}

A amostra foi composta por estudantes da Universidade Federal Rural de Pernambuco (UFRPE), Campus Recife, Pernambuco, Brasil. Foram avaliados 2 grupos: a) grupo treinado (GT) , submetido a 8 semanas de treinamento aeróbio de intensidade moderada $(n=8)$ e b) grupo controle (GC), que não foi submetido a qualquer intervenção $(n=8)$.

A abordagem foi realizada através da colocação de flyers, após licença da instituição e através das redes sociais da universidade, a fim de divulgar os objetivos da pesquisa e selecionar voluntários que os critérios de inclusão do estudo. A amostragem foi definida por conveniência e a alocação dos grupos feita de forma aleatória. 
Foram incluídos no estudo indivíduos saudáveis de 18 a 37 anos, estudantes de qualquer curso da UFRPE e sedentários. Foram excluídos do estudo todos os indivíduos que apresentavam alguma doença pré-existente que pudesse impedir a prática do treinamento físico ou que apresentassem incapacidade cognitiva para responder aos questionários.

Para a avaliação foram utilizados três questionários: o questionário internacional de atividade física - versão curta (IPAQ - versão curta $)^{15}$, o índice de qualidade do sono de Pittsburgh (PSQI) ${ }^{16}$ e a escala de sonolência de Epworth (ESE) ${ }^{16}$.

O IPAQ - versão curta ${ }^{15}$ avalia a frequência e a duração das atividades físicas realizadas pelos indivíduos durante a semana, classificandoas em três tipos: caminhada, atividades físicas moderadas e atividades físicas vigorosas. Depois de avaliar as respostas, classifica os indivíduos em: sedentários, irregularmente ativos, ativos e muito ativos. São considerados sedentários os indivíduos que não realizam nenhuma atividade física por pelo menos 10 minutos contínuos durante a semana.

O PSQI é composto por 10 questões e avalia os 7 componentes a seguir: componente 1: qualidade subjetiva do sono; componente 2: latência do sono; componente 3: duração do sono; componente 4: eficiência habitual do sono; componente 5: distúrbios do sono; componente 6: uso de medicação para dormir e componente 7 : disfunção durante o dia. A pontuação dos sete componentes somados varia de 0 a 21 . A avaliação acontece da seguinte forma: pontuações até 5 indicam uma qualidade do sono boa (são considerados bons dormidores) e pontuações acima de 5 indicam uma qualidade do sono ruim (são considerados maus dormidores) ${ }^{16}$.

A ESE é baseada em 8 situações do cotidiano, e o indivíduo avaliado responde qual a probabilidade de cochilar ou adormecer nessas situações. A pontuação para cada resposta varia de 0 a 3 , sendo 0 , nenhuma chance, 1 , pequena chance, 2, chance moderada e 3 alta chance de cochilar. A soma das oito situações do cotidiano pode alcançar 24 pontos. A avaliação da sonolência diurna excessiva é: $<10$ : normal e $\geq 10$ : presença de sonolência diurna excessiva ${ }^{16}$.

Os questionários foram preenchidos em uma sala reservada, na presença de, no mínimo, 1 (um) pesquisador para cada 2 entrevistados em tempo estimado de 30 minutos, respeitandose os direitos de privacidade e confidencialidade dos dados coletados.

O estudo foi aprovado pelo Comitê de Ética e Pesquisa da Faculdade Integrada do Recife, conforme a resolução 466/2012 do Conselho Nacional de Saúde (CAAE: 0008.0.100.000-10). Os participantes tinham o direito de retirar-se do estudo a qualquer momento, sem qualquer penalidade.

Ao todo foram recrutados 28 candidatos: 2 foram excluídos por não cumprirem os critérios de elegibilidade e 10 por não completarem a fase de treinamento. No total, 16 voluntários completaram todas as etapas da pesquisa (Figura 1).

Os voluntários do grupo treinado foram submetidos a um teste de esforço máximo em esteira (Protocolo de Ellestad), com o objetivo de determinar o nível de aptidão cardiorrespiratória. Antes do início do teste, os voluntários permaneceram em pé na esteira, por aproximadamente 2 minutos, para avaliação e registro do eletrocardiograma (ECG) de 12 derivações de repouso com o programa de computador Ergo PC para Windows (MICROMED ${ }^{\circledR}$ Biotecnologia), e avaliação da pressão arterial (PA) em repouso, através do método auscultatório. Durante a realização do teste e nos primeiros seis minutos de recuperação, foram realizados registros contínuos do ECG e medidas auscultatórias de PA (a cada 3 minutos durante o exercício e no pico de esforço e a cada minuto de recuperação). $\mathrm{O}$ teste foi interrompido quando o paciente relatou exaustão, atingiu a frequência cardíaca máxima ou apresentou quaisquer sinais ou sintomas.

Através dos dados obtidos durante a realização do teste ergométrico (TE), a medida indireta do consumo máximo de oxigênio $\left(\mathrm{VO}_{\text {máx }}\right)$ 


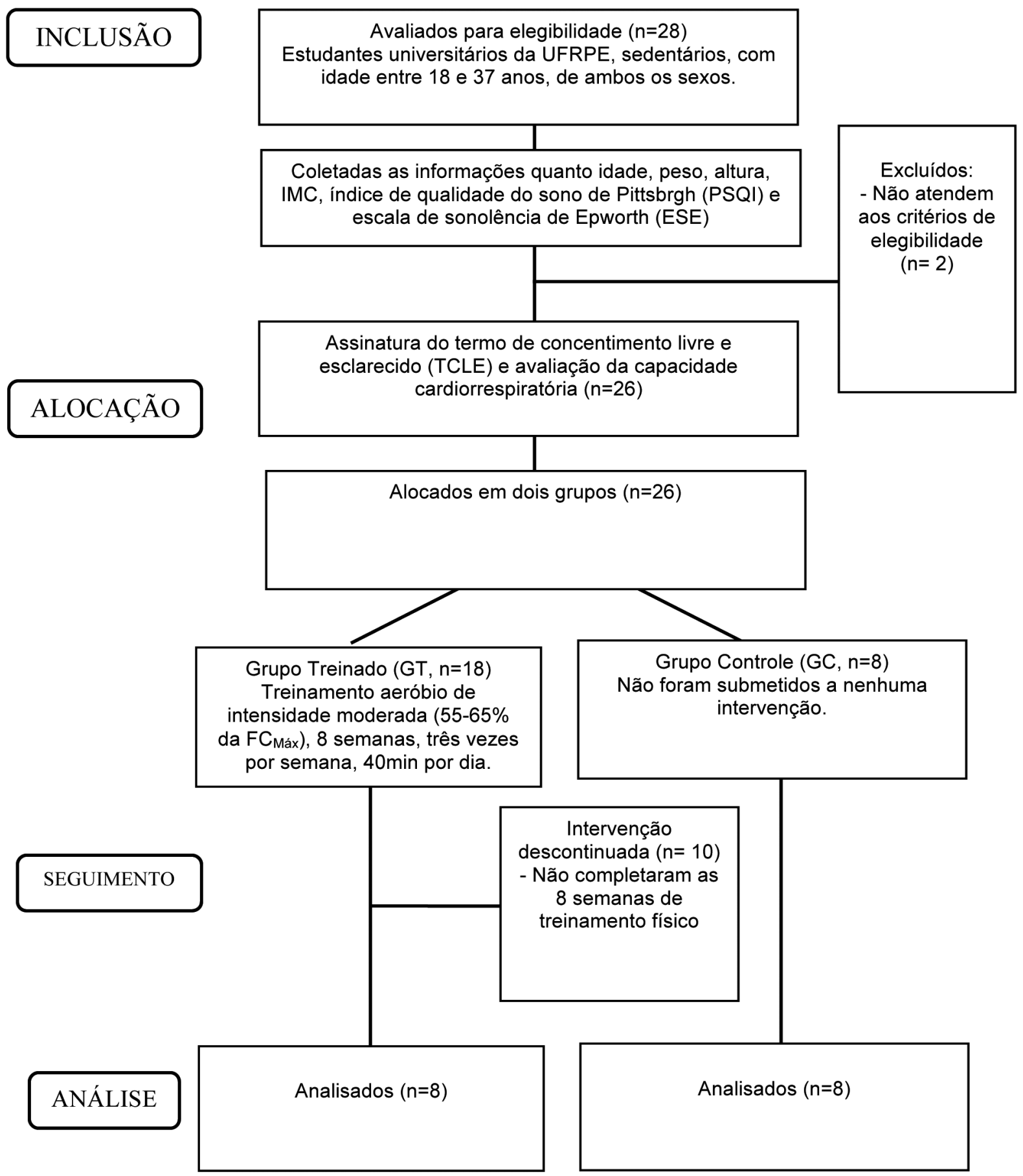

Figura 1: Fluxograma da sequência experimental.

Fonte: os autores.

em $\mathrm{ml} / \mathrm{Kg} / \mathrm{min}$ foi calculada automaticamente pelo programa de computador.

Para o treinamento do GT, foi estabelecida um zona entre 55 e $65 \%$ da freqência cardíaca máxima dos voluntários. O cálculo foi realizado através da equação de Karvonen (FCT $=\mathrm{FCR}+\%$ $($ FCM - FCR)), onde: FCT = frequência cardíaca de treinamento; FCR = frequência de repouso; $\mathrm{FCM}=$ frequência cardíaca máxima e $\%=$ intensidade do treino. Durante todo treinamento 
os voluntários tiveram sua frequência cardíaca monitorada por um frequencímetro com precisão de um batimento por minuto.

No GT, o protocolo de treinamento aeróbio tinha duração de 40 minutos por sessão, três vezes por semana, num total de 8 semanas, em uma bicicleta ergométrica. Previamente à realização do exercício, ocorria uma sessão de alongamento com duração média de 5 a 10 minutos, para preparar a musculatura das principais articulações de membros superiores, inferiores e região do tronco. A qualidade do sono e a sonolência diurna foram avaliadas antes e após a realização do protocolo de 8 semanas de treinamento.

O GC não realizou o protocolo de treinamento, com isso teve sua qualidade do sono e sonolência diurna excessiva avaliadas duas vezes em um intervalo de 8 semanas.

Para análise dos dados foi utilizada estatística inferencial através do Software SPSS Versão 20.0. Para comparação intragrupo da qualidade do sono e sonolência diurna excessiva, antes e após as 8 semanas de treinamento, utilizamos o teste $\mathrm{t}$ Student para amostras dependentes e, para comparação intergrupos das mesmas variáveis, utilizamos o teste $t$ Student para amostras independentes. Os resultados estão mostrados como média \pm desvio padrão e foi considerado um p $<0,05$ como nível de significância estatística.

\section{Resultados}

Na tabela 1, podemos observar as caraterísticas basais de sexo, idade, altura, peso e índice de massa corporal (IMC) de ambos os grupos, não havendo diferença entre eles.

Na figura 2, podemos observar os dados referentes à qualidade do sono. Na comparação intergrupos, o GT apresentou um menor escore do PSQI comparado ao grupo controle (controle: $6,2 \pm 2,3$ vs. treinado: $3,7 \pm 1,9 ; p<0,03$ ). Além disso, na comparação intragrupos, o GT melhorou a qualidade do sono (pré: $8,6 \pm 3,7$ vs. pós: $3,7 \pm 1,9$; $\mathrm{p}<0,05)$, enquanto o GC não mostrou nenhuma alteração nesta variável (pré: $6,1 \pm 2,7$ vs. pós: 6,2 $\pm 2,3 ; \mathrm{p}=0,76$ ).

Na figura 3, verificamos que sobre a SDE, na comparação intragrupos, não houve alteração no GT (pré: $7,6 \pm 3,5$ vs. pós: $6,8 \pm 2,2 ; p=0,45$ ), nem no GC (pré: $11,8 \pm 5,6$ vs. pós: $12,0 \pm 4,0 ; p=0,91$ ).

Na tabela 2, pode ser observado que, antes do treinamento físico, 7 (87,5\%) voluntários do GT, apresentaram qualidade de sono ruim. Após a intervenção, todos os voluntários melhoraram

Tabela 1: Características da Amostra

\begin{tabular}{ccc}
\hline & $\begin{array}{c}\text { Grupo Controle } \\
(\mathrm{n}=8)\end{array}$ & $\begin{array}{c}\text { Grupo Treinado } \\
(\mathrm{n}=8)\end{array}$ \\
\hline Idade (anos) & $21,2 \pm 4,1$ & $21,0 \pm 1,8$ \\
\hline Sexo $(\mathrm{M} / \mathrm{F})$ & $1 / 7$ & $5 / 3$ \\
\hline Peso $(\mathrm{kg})$ & $58,3 \pm 9,2$ & $74,0 \pm 13,6$ \\
\hline Altura $(\mathrm{m})$ & $1,63 \pm 0,07$ & $1,68 \pm 0,10$ \\
\hline IMC $\left(\mathrm{kg} / \mathrm{m}^{2}\right)$ & $21,8 \pm 3,9$ & $26,3 \pm 3,7$ \\
\hline PSQI & $6,1 \pm 2,7$ & $8,6 \pm 3,7$ \\
\hline ESE & $11,9 \pm 5,4$ & $7,6 \pm 3,5$
\end{tabular}

IMC = Índice de Massa Corpórea; PSQI: Índice de Qualidade do Sono de Pittsburgh; ESE: Escala de Sonolência de Epworth. Os resultados da tabela estão expressos em média \pm desvio padrão.

Fonte: os autores

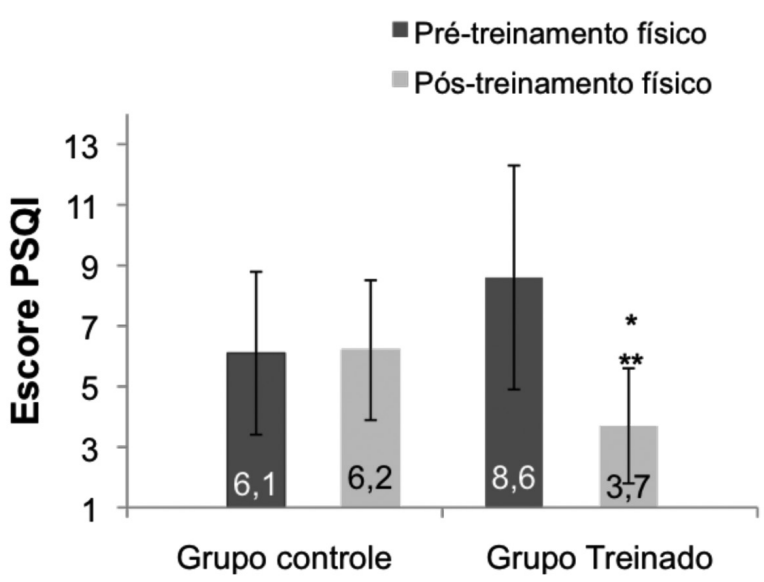

Figura 2: Comparação dos escores do PSQI do grupo treinado e controle antes e após 8 semanas de treinamento

${ }^{*} p<0,05$ grupo controle pré vs. grupo controle e grupo treinado pré vs. grupo treinado pós; ${ }^{* *} p<0,05$ grupo treinado pós vs. grupo controle pós. PSQI: índice de qualidade do sono de Pittsburgh Fonte: Os autores. 
- Pré-treinamento físico

-Pós-treinamento físico

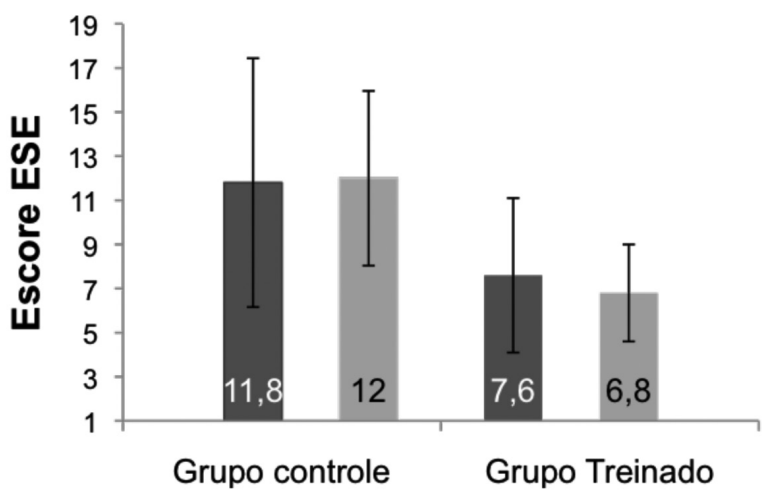

Figura 3: Comparação dos escores da ESE do grupo treinado e controle antes e após as 8 semanas de treinamento

* $p<0,05$ grupo controle pré vs. grupo controle e grupo treinado pré vs. grupo treinado pós

Fonte: os autores.

sua pontuação, mas apenas $6(75 \%)$ atingiram pontuação suficiente para ter um sono considerado adequado. Antes do treinamento, 7 (87,5\%) voluntários do GT não apresentaram nenhum grau de sonolência diurna excessiva, não sendo constatada nenhuma mudança na quantidade de indivíduos sem sonolência, após a realização do treinamento.
Já no GC, não houve mudanças na quantidade de indivíduos que apresentavam qualidade do sono ruim após as 8 semanas de treinamento. Com relação à sonolência diurna excessiva, antes do treinamento, 5 (62,5\%) voluntários apresentavam sonolência diurna excessiva e após as 8 semanas de treinamento, $6(75 \%)$ deles apresentaram este sintoma.

\section{Discussõ̃o}

Os nossos resultados demonstraram que o treinamento aeróbio de intensidade moderada durante 8 semanas, quando aplicado de forma regular em estudantes universitários sedentários, foi eficaz na melhoria da qualidade do sono destes indivíduos, apesar de não afetar a sonolência diurna excessiva.

No nosso estudo, no GT, antes de ser submetido ao treinamento aeróbio de intensidade moderada, apenas $12,5 \%$ dos voluntários apresentavam qualidade do sono dentro dos padrões da normalidade. Segundo Almeida et al. ${ }^{17}$, a qualidade do sono e seus distúrbios em estudantes têm sido bastante pesquisados nos últimos anos. A preocupação com essa temática pode estar relacionada ao fato do sono ser um elemento fundamental na

Tabela 2: Resultado clínico do número e percentutal de pacientes dos grupos controle e treinado que apresentaram melhora, não alteração ou piora do índice de qualidade do sono de Pittsburgh (PSQI) e escala de sonolência de Epworth (ESE).

\begin{tabular}{lcccc}
\hline & \multicolumn{2}{c}{ Grupo Controle $(\mathrm{n}=8)$} & \multicolumn{2}{c}{ Grupo Treinado $(\mathrm{n}=8)$} \\
\cline { 2 - 5 } & Antes de 8 semanas & Após 8 semanas & Pré Treinamento & Pós Treinamento \\
\hline PSOI & & & & \\
$\leq 5$ (Bons Dormidores) & $3,66 \pm 1,52$ & $4,33 \pm 0,57$ & $5 \pm 0$ & $2,83 \pm 0,98$ \\
& $(n=3 ; 37,5 \%)$ & $(n=3 ; 37,5 \%)$ & $(n=1 ; 12,5 \%)$ & $(n=6 ; 75 \%)$ \\
$>5$ (Maus Dormidores) & $7,6 \pm 2,07(n=5 ;$ & $7,85 \pm 2,19$ & $9,14 \pm 3,71$ & $6,5 \pm 0,70$ \\
& $62,5 \%)$ & $(n=5 ; 62,5 \%)$ & $(n=7 ; 87,5 \%)$ & $(n=2 ; 25 \%)$ \\
\hline ESE & & & & \\
$<10$ (Ausência de & $6 \pm 1$ & $6,5 \pm 3,53$ & $6,71 \pm 2,62$ & $6,42 \pm 1,98$ \\
$\quad$ Sonolência) & $(n=3 ; 37,5 \%)$ & $(n=2 ; 25 \%)$ & $(n=7 ; 87,5 \%)$ & $(n=7 ; 87,5 \%)$ \\
210 (Presença de & $15,4 \pm 3,71$ & $13,8 \pm 1,83$ & $14 \pm 0$ & $10 \pm 0$ \\
Sonolência) & $(n=5 ; 62,5 \%)$ & $(n=6 ; 75 \%)$ & $(n=1 ; 12,5 \%)$ & $(n=1 ; 12,5 \%)$ \\
\hline
\end{tabular}

PSQI: Índice de Qualidade do Sono de Pittsburgh; ESE: Escala de Sonolência de Epworth. Os resultados da tabela estão expressos em média desvio padrão; (n/\%)

Fonte: os autores. 
vida dos estudantes, influenciando diretamente na qualidade de vida, o que impacta diretamente na saúde e nas atividades acadêmicas.

Nossos resultados diferem dos encontrados por Almeida et al $^{17}$, que em seu estudo avaliaram a qualidade do sono em estudantes de fisioterapia. Eles demonstraram que 52\% da amostra pesquisada apresentaram qualidade do sono dentro dos padrões da normalidade. Os resultados encontrados por Almeida et al. ${ }^{17}$ são semelhantes aos observados por Trindade-Filho et al. ${ }^{18}$ e Furlani e Ceolim ${ }^{19}$. Já Araújo et al..$^{20}$, em seu estudo com estudantes universitários de Fortaleza$\mathrm{CE}$, constataram que praticamente toda a amostra analisada era de maus dormidores. Carvalho et al. ${ }^{21}$, avaliando estudantes universitários das áreas de saúde e ciências exatas, verificaram que tanto os estudantes de saúde como os estudantes de exatas apresentaram, em sua maioria, uma má qualidade do sono. $81,48 \%$ dos estudantes da área de saúde e $65,22 \%$ da área de exatas foram classificados com qualidade do sono ruim. Pascotto e Santos ${ }^{22}$, no seu estudo com estudantes universitários, identificaram $61,8 \%$ da amostra com uma má qualidade do sono, resultados que se aproximam mais aos encontrados na nossa pesquisa. Araújo et al..$^{20}$ atribuem essa discrepância dos resultados à não randomização na seleção dos sujeitos. Assim, é possível que os voluntários que já sabiam ou desconfiavam ter problemas de sono, tenham tido mais interesse em participar do estudo. Fato semelhante pode ter acontecido no nosso estudo, por se tratar de uma pesquisa na qual havia uma intervenção e tinha como objetivo verificar se o exercício era eficaz para melhorar a qualidade do sono.

Após o treinamento, $75 \%$ dos voluntários atingiram pontuação suficiente para serem considerados bons dormidores. Este resultado corrobora com os estudos de Vuori et al. ${ }^{23}$ e Sherrill et al. ${ }^{24}$. Estes autores afirmam que exercícios de intensidade moderada e executados de forma regular promovem benefícios sobre a qualidade de sono de seus praticantes. Martins et al. ${ }^{10}$ relatam que os exercícios físicos de duração e intensidades diferentes podem auxiliar no tra- tamento e prevenção de alguns distúrbios do ciclo sono-vigília, seja diretamente, por diminuir a fragmentação do sono, provocar aumento no sono de ondas lentas e reduzir da latência para início do sono ou, indiretamente, através do controle de peso e aquisição de hábitos saudáveis.

Alguns estudos tem procurado explicar os benefícios do exercício físico sobre a melhora do padrão do sono, e se apoiam em três hipóteses: a primeira, conhecida como termorregulatória, sugere que o disparo para o início do sono seja o declínio da temperatura do corpo durante a noite, que é gerado primeiramente pelo aumento da circulação periférica que dissipa calor ${ }^{25}$. $\mathrm{O}$ exercício físico aumenta a temperatura corporal proporcionalmente à carga de trabalho ${ }^{26}$. Por exemplo, um indivíduo se exercitando em $70 \%$ do consumo máximo do oxigênio $\left(\mathrm{VO}_{2 \mathrm{MÁx}}\right)$, elevaria a temperatura corporal em aproximadamente $2^{\circ} \mathrm{C}$, a partir do 15-20 minutos de trabalho contínuo, com uma carga de trabalho constante e duração de 60 minutos. Portanto, esta hipótese afirma que o aumento da temperatura corporal, como consequência do exercício físico, facilitaria o disparo do início do sono, graças à ativação dos mecanismos de dissipação do calor e de indução do sono, através de neurônios termosensíveis controlados pelo hipotálamo ${ }^{27,28}$.

Sobre a segunda hipótese, conhecida como conservação de energia, é afirmado que o aumento do gasto energético promovido pelo exercício durante a vigília aumentaria a necessidade de sono. Justifica-se este fato pela ação do sono na redução da taxa metabólica, quando comparado ao repouso em vigília, a fim de alcançar um balanço energético positivo (homeostase), restabelecendo uma condição adequada para um novo ciclo de vigília ${ }^{27}$. Já a terceira hipótese, restauradora ou compensatória, da mesma forma que a anterior, relata que a alta atividade catabólica durante a vigília e, sobretudo no exercício, reduz as reservas energéticas, aumentando a necessidade de sono e favorecendo a atividade anabólica ${ }^{27}$. Reid et $\mathrm{al}^{29}$, estudando idosos com insônia crônica, identificaram que o exercício físico aeróbio moderado funcionou como um 
tratamento eficaz para melhorar a qualidade do sono, humor e qualidade de vida e diminuir o nível de sonolência diurna excessiva dos participantes da pesquisa. Yang et al. ${ }^{30}$ afirmam que a participação em um programa de treinamento de exercícios aeróbicos de intensidade moderada ou exercícios resistidos de alta intensidade tem efeitos positivos sobre a qualidade do sono em adultos de meia idade e mais velhos e que o exercício físico pode ser uma abordagem alternativa ou complementar às terapias farmacológicas existentes para problemas de sonoMello et al. ${ }^{14}$, em seu estudo realizado na cidade de São Paulo, fez um levantamento e demonstrou que entre $27,1 \%$ e $28,9 \%$ de pessoas fisicamente ativas e $72,9 \%$ e $71,1 \%$ entre os sedentários se queixavam de insônia e sonolência excessiva, respectivamente. No nosso estudo, 33,3\% dos voluntários antes do treinamento apresentaram nível de sonolência dentro dos padrões da normalidade, 33,3\% apresentaram nível de sonolência considerado no limite e $27,7 \%$ apresentaram sonolência leve. Apesar de não ter havido melhora na sonolência diurna excessiva dos participantes após o treinamento aeróbio, a maioria dos voluntários apresentaram o nível de sonolência diurna dentro dos padrões da normalidade e a minoria apresentou resultados considerados limítrofes, de acordo com os escores obtidos na ESE.

Almeida et al. ${ }^{17}$ afirmam que a sonolência diurna excessiva é um distúrbio que interfere tanto na qualidade de vida, quanto no desempenho cognitivo, atencional, profissional e no relacionamento social, aumentando os riscos de acidentes de trabalho e de trânsito. Connor et al. ${ }^{31}$, em seu estudo, afirmam que a proporção de acidentes de trânsito que pode estar relacionada com a SDE nos Estados Unidos é entre 1\% a 3\% e, na Austrália, de 33\%.

O nosso estudo apresenta algumas limitações. Primeiramente, no que diz respeito ao caráter metodológico do estudo, pois não utilizamos randomização na seleção dos sujeitos. A realização de um ensaio clínico randomizado, traria maior rigor metodológico ao estudo. Além disso, a análise objetiva da qualidade do sono através a actigrafia, poderia fornecer dados mais fidedignos sobre o perfil do sono dos voluntários. A actigrafia é um método de avaliação do ciclo sono-vigília que permite o registro da atividade motora através dos movimentos dos membros durante 24 horas. Desta forma, são obtidas informações como tempo total de sono, tempo total acordado, número de despertares e latência para o início do sono.

\section{Conclusões}

De acordo com os resultados do presente estudo, podemos concluir que o treinamento físico aeróbio entre de intensidade moderada melhora a qualidade do sono, mas parece não alterar a sonolência diurna excessiva dos estudantes universitários.

Grande parte dos estudantes universitários envolvidos no estudo apresentaram uma qualidade do sono ruim e algum grau de sonolência diurna excessiva. Isto pode estar relacionado com os maus hábitos adquiridos ao longo da vida ou com as demandas acadêmicas da universidade. Sugere-se, portanto, a adoção de ações estruturadas no meio acadêmico, através de programas ou projetos destinados a este público, visando a melhora da qualidade do sono, estimulando a prática de exercícios físicos regulares para os estudantes universitários.

\section{Agradecimentos}

Agradecemos a CnPQ/UFRPE pelo apoio finaceiro através do Programa Institucional de Bolsas de Iniciação Científica.

\section{Referênciass}

1. Hill VM, O'Connor, RM, Shirasu-Hiza, M. Tired and stressed: Examining the need for sleep. Eur J Neurosci. 2018 (citado 2018 Nov 10]; 8. https://doi. org/10.1111/ejn.14197. 
2. Roeths $\mathrm{T}$, Roth T. Sleep-wake state and memory function. Sleep. 2000; 23: 64-8.

3. Da Costa SV, Ceolim MF. Fatores que interferem na qualidade do sono de pacientes internados. Rev da Esc de Enferm da USP. 2013; 47(1): 46-52.

4. Abrams, RM. Sleep Deprivation. Obstet Gynecol Clin North Am. 2015; 42:493-506.

5. Aloé F, Silva AB. Sono Normal e Polissonografia. In: PINTO, J.A. Ronco e Apnéia do Sono. Rio de Janeiro, Rewinter. 2000; 3: 9-16.

6. Thorpy, MJ. Classification of sleep disorders. Neurotherapeutics. 2012; 9:687-701.

7. Bonnet MH, Arand, DL. Clinical effects of sleep fragmentation versus sleep deprivation. Sleep Med Rev. 2003; 7(4): 297-310.

8. Brouwers FM, Lenders JW, Sleep-disordered breathing and hypertension. N Engl J Med. 2000; 343 (13): 967.

9. Mello MT, Santana MG, Souza LM, Oliveira PCS, Ventura ML, Stampi C. Sleep patterns and sleeprelated complaints of Brazilian inter-state bus drivers. Braz J Med Biol Res. 2000; 33(1): 71-7.

10. Martins PJF, Mello MT, Tufik S. Exercício e sono. Rev Bras Med Esporte. 2000; 7 (1): 28-36.

11. Sateia MJ. International classification of sleep disorders-third edition: highlights and modifications. Chest. 2014 Nov;146(5):1387-1394.

12. Santiago LS, Lyra MJ, Cunha-Filho M, Cruz, PWS, Santos MAM, Falcão, APST. Efeito de uma sessão de treinamento de força sobre a qualidade do sono de adolescentes. Rev Bras Med Esporte. 2015; 21(2): 148-152.

13. Steffens RAK, Liz CM, Viana MS, Brandt R, Oliveira LGA, Andrade, A. Praticar caminhada melhora a qualidade do sono e os estados de humor em mulheres com síndrome da fibromialgia. Rev Dor. 2011; 12(4): 327-31.

14. Mello TM, Fernandez AC, Tufik S. Levantamento epidemiológico da prática de atividade física na cidade de São Paulo. Rev Bras Med Esporte. 2000; 6:119-24

15. Matsudo S, Araújo T, Matsudo V, Andrade D, Andrade E, Braggion G. Questionário internacional de atividade física (IPAQ): estudo de validade e reprodutibilidade no Brasil. Rev Bras Ativ Fís Saúde. 2001;05-18
16. Bertolazi AN. Tradução, adaptação cultural e validação de dois instrumentos de avaliação do sono: escala de sonolência de Epworth e índice de qualidade de sono de Pittsburgh [Dissertação de mestrado]. Porto Alegre: Universidade Federal do Rio Grande do Sul; 2008.

17. Almeida JOS, Simões de Siqueira PP, Lima AMJ, Brasileiro-Santos MDS, Galindo Filho VC. Sonolência diurna e qualidade do sono em estudantes universitários de fisioterapia. Rev ConScientiae e Saúde. 2011; 10(4): 201-07

18. Trindade-filho EM, Carvalho LNA, Gomes EO. Estudo da qualidade do sono na população adulta de Maceió. Neurobiologia. 2010; 73 (1): 93-97.

19. Furlani R, Ceolim MF. Padrões de sono de estudantes ingressantes na Graduação em Enfermagem. Rev Bras Enferm. 2005; 58 (3): 320-4.

20. Araújo MFM, Lima ACS, Alencar AMPG, De Araújo TM, Fragoaso LVC, Damasceno MMC. Avaliação da qualidade do sono de estudantes universitários de Fortaleza-CE. Texto \& contexto enfermagem, 2013; 22(2): 352-360.

21. Carvalho TMCS, Silva Junior II, Siqueira PPS, Almeida JO, Soares AF, Lima AMJ. Qualidade do sono e sonolência diurna entre estudantes universitários de diferentes áreas. Rev Neurociênc, 2013; 21(3): 383-7.

22. Pascotto AC, Santos BRM. Avaliação da qualidade do sono em estudantes de ciências da saúde. J Health Sci Inst. 2013; 31(3): 306-310.

23. Vuori, I, Urponen H, Hasan J, Partinen, M. Epidemiology of exercise effects on sleep. Acta Physiol Scand Suppl. 1987; 574: 3-7.

24. Sherrill DL, Kotchou K, Quan SF. Association of physical activity and human sleep disorders. Arch Intern Med. 1998; 158 (17): 1894-1898.

25. Murphy PJ, Campbell SS. Nighttime drop in body temperature: a physiological trigger for sleep onset? Sleep 1997; 20: 505-511

26. Saltin B, Hermansen L. Esophageal, rectal, and muscle temperature during exercise. J Appl Physiol 1966; 21: 1757-1762

27. Driver HS, Taylor S. Exercise and sleep. Sleep Med Rev, 2000; 4: 387-402

28. Lu J, Greco MA, Shiromani P, Saper CB. Effects of lesions of the ventrolateral preoptic nucleous on NREM and REM sleep. J Neurosci. 2000; 20: 3830-42

29. Reid KJ, Baron KG, Lu B, Naylor E., Wolfe L, Zee PC. Aerobic exercise improves self-reported sleep and quality of life in older adults with insomnia. Sleep medicine. 2010; 11 (9): 934-940. 
30. Yang PY, Ho KH, Chen HC, Chien MY. Exercise training improves sleep quality in middle-aged and older adults with sleep problems: a systematic review. J Physiother. 2012; 58 (3): 157-163.
31. Connor J, Norton R, Ameratunga S, Robinson E, Civil I, Dunn R, et al. Driver sleepiness and risk of serious injury to car occupants: population based case control study. BMJ. 2002; 324 (7346): 1125. 